\title{
Téoros
}

Revue de recherche en tourisme

\section{Paysages du littoral}

La frange extensible (du pays métissien)

\section{Philippe Poullaouec-Gonidec, François Tremblay et Christiane Montpetit}

Volume 20, numéro 1, printemps 2001

Mers et littoraux

URI : https://id.erudit.org/iderudit/1071910ar

DOI : https://doi.org/10.7202/1071910ar

Aller au sommaire du numéro

Éditeur(s)

Université du Québec à Montréal

ISSN

0712-8657 (imprimé)

1923-2705 (numérique)

Découvrir la revue

Citer cet article

Poullaouec-Gonidec, P., Tremblay, F. \& Montpetit, C. (2001). Paysages du littoral : la frange extensible (du pays métissien). Téoros, 20(1), 41-48.

https://doi.org/10.7202/1071910ar d'utilisation que vous pouvez consulter en ligne.

https://apropos.erudit.org/fr/usagers/politique-dutilisation/ 


\section{Paysages du littoral La frange extensible (du pays métissien)}

\section{Philippe Poullaouec-Gonidec, François Tremblay et Christiane Montpetit}

Monologue à propos de la difficulté de peindre la mer pour un peintre de modèle vivant :

" La mer, c'est difficile. [...] Le probleme, c'est : où diable peuvent-ils bien être les yeux de la mer? Je $n$ 'arriverai à rien tant que je n'aurai pas découvert ça, parce que c'est là le commencement, comprenezvous? le commencement de tout, et tant je n'aurai pas compris où est le commencement, je continuerai à passer mes journées à regarder cente mandite étendue d'can [... J. * (Baricco, 1993 : 94)

Dialogue (en réponse au premier monologue):

$Q:$ Où ils sont, les yeux de la mer? [...]

$R$ : Les bateaux sont les yeux de la mer [...J Et elle, elle a des centaines d'yeux. Vous ne voudriez quant même pas qu'elle doive se débrouiller avec deux [...]

$Q:$ Et les naufrages? Les tempetes, les typhons, toutes ces choses [...] Pourquoi avaleraitelle tous ces bateaux, si c'était ses yeux ? [...]

$R:$ Ef vous $[\ldots]$ wous ne les fermez jamais, wos yeux? (Baricco, 1993 : 102-103)
D epuis plus d'une quinzaine d'années, le fleuve Saint-Laurent réapparaît comme une des figures paysagères les plus importantes au Québec. Qu'il s'agisse de la mise en valeur de ses îles ou de l'interprétation du transport maritime, on constate un retour du fleuve au centre des relations esthétiques que la société québécoise entretient à l'endroit de son territoire. A la faveur de cette redécouverte, répparaissent aussi certaines valorisations des formes territoriales associées au fleuve. Ces formes territoriales associées au SaintLaurent comprennent autant les artefacts culturels, c'est-à-dire les phares, les aboiteaux, la structure rangique ou les traces archéologiques basques, que les éléments physiographiques tels que les monadnocks de Kamouraska, les murailles de Saint-Fabien ou les collines du Bic. Audelà de ces valorisations d'éléments ponctuels, on constate également une reconnaissance de la côte en tant qu'entité paysagère qu'il faut mettre en valeur, au moins visuellement, pour les visiteurs et les touristes. Par exemple, la a Route des Navigateurs s et l'inscription de la route 132 aux schémas d'aménagement en tant que route panoramique représentent autant d'initiatives qui témoignent que la côte de notre fleuve commence à être considérée comme potentiellement porteuse de pay sages uniques et singuliers.

Malgré un tel foisonnement des préoccupations paysagères à l'endroit du fleuve et des éléments associés, on constate l'absence d'une réflexion d'ensemble concernant le littoral en tant que forme territoriale globale qui suscitent des appréciations paysagères spécifiques. En effet, derrière la figure paysagère du fleuve se profile un phénomène territorial riche en significations paysagères : le littoral comme espace linéaire de contact entre deux réalités élémentaires. C'est la rencontre de la terre et de l'eau (la mer et le fleuve). Ces formes élémentaires révèlent toutefois des substrats imaginaires contrastés et complexes par leur nature. Lieu, environnement et cadre de vic, la terre est notre référent. Elle appartient à l'univers du domestiqué. L'étendue a fleuve/mer *, pour sa part, est l'espace de la distanciation que l'on côtoie à bras le corps occasionnellement par la baignade, par la voile ou par métier, mais qui reste avant tout l'espace des représentations.

Le * fleuve/mer * n'est que mouvance. Conjonction de marées, de courants et de vents, sa structure nous est livrée par les vagues, leur miroitement, leur grondement ou leur clapotis. Aucune trace ne peut s'y fixer et sa surface sans cesse tourmentée laisse paraître l'angoisse d'un tréfonds. Il est bon de se rappeler que seule la mer est un état brut qui n'a pas changé depuis la nuit des temps. Les bateaux et les baleines au large de la côte ne sont que des passages furtifs et, parfois, des rendez-vous attendus.

Plus que tout, et en comparaison avec la terre, la couleur de l'étendue de l'eau et ses ambiances de jour et de contre-jour sont intimement liées à l'état du ciel, aux dessins des nuages et à leurs ombrages sur l'eau. Ces caractéristiques sont si prégnantes qu'elles fascinent le spectateur. Perché sur un rocher ou marchant sur la grève, le spectateur du rivage est en constante quête d'un morceau d'arbre échoué, d'imprévu ou d'un possible imaginé, comme la ve- 
nue d'une déferlante monstrueuse ou d'un bateau naufrage, pour assouvir une sublimation, à l'image du tableau La tempête du peintre Joseph Vernet $(1777)^{1}$.

Cette étendue ne livre aucunement les traces matérialisées d'un passé. Ces traces sont imaginaires. Au-delà du simple spectacle, la mer renferme en elle tous les passés. Le paysage marin ne peut être évoqué qu'à travers le récit ou lạ peinture, ou encore capté l'instant d'une photographie, d'un bout de film. Rien, absolument rien, ne permet d'illustrer les éléments d'un récit passé. Seule sa rencontre avec la terre permet au littoral d'évoquer quelques histoires. Les jetees, les phares en bout de pointe, les fascines, les bouées de mouillage, les échouages sont les principaux fragments d'une histoire à décrypter. Le littoral est en quelque sorte la rencontre superposée de deux réalités dissociées que chaque partie cherche à gagner sur l'autre.

La mer efface, la nuit (a propos des traces de pas dans le sable). La maree recouvre. Comme si personne n'était jamais passé. Comme si nous n'avions jamais existé. S'il y a, dans le monde, un endroit où tu peur penserque tu n'es rien, cet endroit, c'est ici. Ce n'est pas encore la terre, et ce n'est pas encore la mer. Cen'est pas whe vie fausse, et ce n'est pas une vie vraie. C'est du temps. Du temps qui passe. Rien d'autre (Baricco, 1993:100).

Le littoral est un phénomène complexe qui a des référents génériques, mais des significations culturelles spécifiques. La réflexion qui est proposee dans les paragraphes suivants aborde l'évolution de cette forme pour dégager des éléments prospectifs relatifs à la mise en paysage du littoral à des fins récréo-touristiques. Pour ce faire, la réflexion est présentée en trois volets : 1) une caractérisation de l'évolution du littoral bas-laurentien ; 2) une analyse des principaux phénomènes qui concourent à la reconnaissance du littoral métissien comme paysage valorisé ; 3) un ênoneé des principaux enjeux qui traversent la mise en valeur des paysages du littoral du pays métissien.

\section{Le littoral bas-laurentien : une forme territoriale en quête de sens}

De manière générale, le littoral est l'espace du lointain, de l'invisible, de l'ailleurs rêvé, imaginé, de l'horizon infini. Le littoral est \& à la fois limite et owverture, aire de résistance et de dissipation. ligne définissante et invitation au vide (White, $1997: 7)$. Il s'agit d'un lieu potentiellement riche en significations. $\mathrm{D}^{+}$autant plus que les ambiances et les prégnances, embruns, airs salins et vents du large, nourrissent ces significations potenticles. Celles-ci peuvent être considérées comme paysagères au sens où elles participent souvent aux processus de production des paysages. Toutefois, ce type de processus est difficile à comprendre parce que complexe. En effet, le processus de production de paysage dépend des relations qu'entretiennent des populations avec leurs territoires. Comme la Chaire l'a proposé au cours d'une récente recherche (PoullaouecGonidec et al., 1999: 11-12), les relations des populations avec le territoire, quelles soient économiques, techniques, symboliques ou spirituelles, conditionnent les relations esthétiques qu'on peut engager avec le territoire ${ }^{2}$ (Berleant, 1997: 148-155). En effet, les interactions des populations avec leur milieu sont fortement porteuses de valeurs identitaires, emblématiques ou affectives qui peuvent participer à la construction de significations paysagères projetées sur les territoires. Par conséquent, les activités qui ont pris place sur ou à proximité du fleuve, à savoir le transport maritime, la pếche, la plaisance ou encore les bains de mer, peuvent faire l'objet de telles valorisations (identitaires, emblématiques et affectives). Il semble donc pertinent de caractériser les activités qui peuvent avoir conditionné les relations entre les populations et le littoral bas-laurentien.

Dans le Bas-Saint-Laurent, l'évolution des logiques qui ont régi les activités sur le fleuve est très marquée. Depuis deux siècles, ces activités ont contribué à construire des significations paysagères spécifiques au littoral bas-laurentien. Cette évolution dans les liens identitaires des populations avec le littoral peut être découpée en trois grands moments :
1-De 1830 à 1950, l'age d'or du trafic maritime longeant la côte sud (Fortin et al., 1993: 484). Durant cette période. le trafic de cabotage joue un rôle important pour les populations locales: les nouvelles du monde arrivent par le fleuve, on va au quai accueillir les pêcheurs, prendre des nouvelles. Parallèlement à cette consolidation du fleuve comme axe est-ouest de transport de marchandises, se développent des lieux très prisés de villégiature qui attirent les bourgeoisies francophones et anglophones. Ce développement d'une villégiature de distinction (Beaudet, 1999 : 154) débute vers la fin du régime seigneurial (1854). Que l'on pense au manoir Rankin-Campbell à Kamouraska ou à l'Estevan Lodge à Grand-Métis, tous deux ont en commun d'avoir été érigés sur des restes de seigneurie. De surcroit, ces deux exemples doivent leur existence à la navigation fluviale : des quais privés de bonnes dimensions étaient construits à proximité. Au cours de toute cette période, le littoral représente l'espace de contact entre la région et des ailleurs multiples ; du plus proche au plus lointain : la ville voisine, les autres lieux de villégiature de l'estuaire, la Gaspésie et I'Europe.

2-De 1940 à 1980, les grandes traversées saisonnières pour le travail (Fortin et al., $1993: 618-628$ ). En raison de la complémentarité des structures économiques entre le Bas-Saint-Laurent et la Côte-Nord, la première étant en situation de surplus de main-d' cuvre, tandis que l'autre doit en importer, on assiste à des migrations saisonnières de la population masculine. Ces migrations $s$ 'effectuent d'abord pour l'exploitation forestière, puis lors de la construction des centrales hydroćlectriques, des complexes Bersimis, Outardes et Manicouagan. Enfin, les grands chantiers d'extraction des minerais dans le Moyen-Nord, de même que les installations de transformation situees sur la Côte-Nord, ont perpétué ces migrations de la main-d'ceuvre jusque dans les années 1980 . Souvent les fils ainés, parfois les maris, doivent quitter la région pour aller travailler durant les 


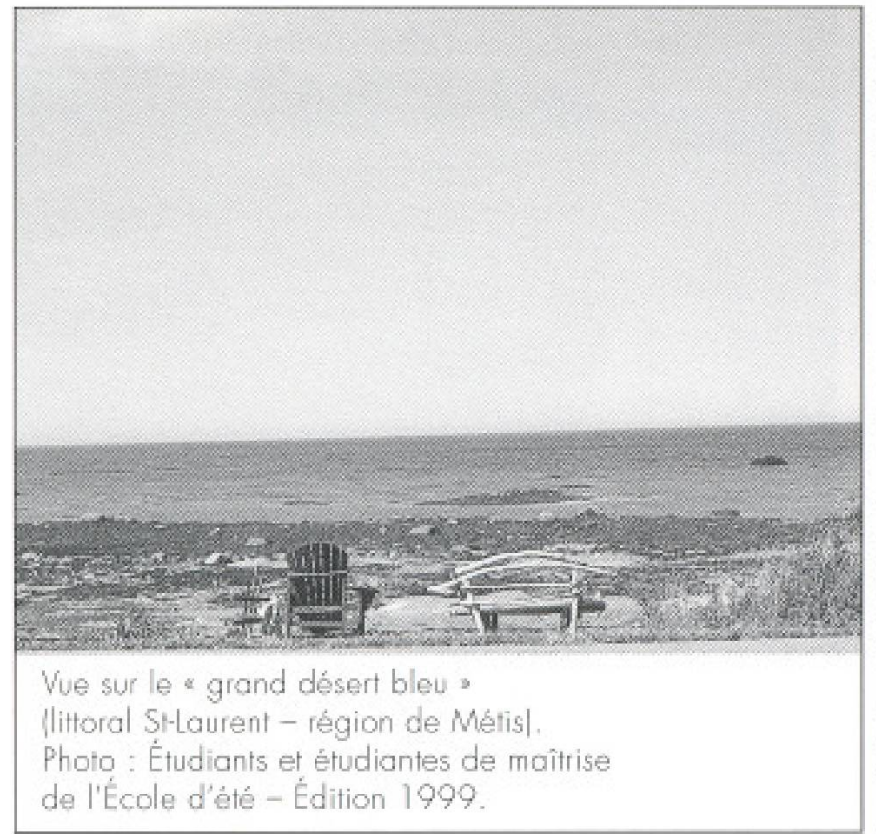

mois d'hiver. Le fleuve devient alors l'étendue qui sépare les familles durant de long mois. Pendant cette période, le littoral représente l'espace de contact entre la région et un ailleurs unique.

\section{3-De 1980 à aujourd hui, le grand desert} bleu. Avec le développement des technologies d'assistance à la navigation et l'abandon progressif du transport maritime de petits tonnages, tout le trafic fluvial longe désormais la Côte-Nord. Le fleuve, a partir du littoral du BasSaint-Laurent, apparait comme une étendue d'eau sur laquelle il n'y a plus aucune activite. A peine perceptibles à l'horizon, les navires marchands glissent en silence sur la voie navigable. Même les voiliers et les barques de peche semblent avoir déserté la côte. Tout est réduit au simple décor, à l'arrière-plan d'un cadrage photographique. La encore, l'ceil du photographe, chasseur d'images spectaculaires, cherche désespếrément le sujet de la photographie.

Cet abandon du fleuve fait en sorte qu'on est désormais devant le $\alpha$ fleuve/mer $\%$ comme devant une ardoise vierge où tout est possible. Depuis 1980 , le littoral du Bas-Saint-Laurent se trouve en quête de sens. Cette quête de sens est comblée partiellement par différentes communautés esthétiques", liés à l'écotourisme, au tourisme culturel ou au tourisme sportif qui, tout en se développant très lentement, proposent des nouveaux sens à ce littoral désormais abandonne.

Cette quête de sens confere au littoral baslaurentien un caractère stratégique dans le contexte d'une mise en paysage à des fins récréotouristiques ${ }^{4}$, En effet, le defi auquel fail face la région du Bas-SaintLaurent au plan touristique consiste à devenit non seulement un lieu de passage vers la Gaspésic, mais egalement un lieu de destination. Dans ce contexte, plusieurs associations locales cherchent à développer et à mettre en valeur les attraits de la région de manière à y retenir les clientèles touristiques. Or, devant un élément territorial aussi fort et présent que le littoral et devant la quête de sens dont il est l'objet, force est de constater que les stratégies touristiques ont intérêt à miser sur les qualités paysagères intrinsèques de ce littoral. Ainsi, en raison de la richesse des significations potentielles que peut supporter le littoral, il y a de fortes possibilités de construction du sens paysager. Pour bien comprendre la porté stratégique d'une réflexion sur la specificité de ce type de paysage, une analyse du littoral métissien semble très pertinente. Cette analyse porte sur les principaux phénomènes qui concourent à la reconnaissance du littoral métissien comme paysage valorise. Il s'agit done de mettre en évidence ce qui, selon nous, semble participer à a l'invention des paysages $x^{5}$ du littoral métissien.

\section{Les paysages du littoral métissien', inventions plurielles}

Comment s'invente le paysage actuel du littoral métissien? Les phénomènes de fabrication du paysage littoral sont toujours pluriels. Dans le cas du pays métissien, soit le littoral s'étendant de Sainte-Flavie à Les Boules, force est de reconnaittre que les inventions paysagères sont relativement récentes et facilement identifiables à un Evénement majeur : la fréquentation de ce coin de pays par la bourgeoisie anglosaxonne à des fins de villégiature. Au-delà de cette invention datée, les paysages du littoral métissien se sont constamment réinventés au cours du $X X^{e}$ siecle. Ces inventions et réinventions sont intimement liées à deux phénomènes : 1) l'invention des paysages par une villegiature qui a connu plusieurs époques de développement : 2) la permanence de l'habitat linéaire et sa reconnaissance en tant que forme paysagère vernaculaire et fortement identitaire.

Il s'agit de deux phenomenes contrastés, puisque l'un émane des regards exogènes, tandis que l'autre provient des regards endogênes. Ces deux phênomènes temoignent à la fois des mutations et des permanences, soit dans les formes territoriales, soit dans les pratiques, soit encore dans le regard de ceux qui fréquentent le territoire. De surcroit, le renouveau du paysage du littoral, tel que l'expriment les deux exemples suivants, provient de l'influence mutuelle des deux types de regard. En effet, au fil du temps, le regard exogène s'est enraciné par la médiation d'un art de jardin, tandis que le regard endogène, par l'émigration et le retour épisodique, en est venu à se distancier du pays.

\section{Métis-sur-Mer : \\ de l'invention à la réinvention du littoral par le jardin}

On peut poser l'hypothèse que le paysage du littoral métissien a été inventé par ceux qui ne vivaient pas de façon permanente dans l'espace littoral et, donc, par ceux qui venaient de l'extérieur (la communauté anglo-saxonne non-résidante de Métis-surMer). Les bateaux blancs, ces navires de croisières touristiques, peuvent être vus comme les instigateurs du regard du villégiateur fortuné (Tittley, 1995). Le rôle du fleuve dans la production de l'espace littoral, par le développement de la villégiature du début du $\mathrm{XX}$ siecle (les grands 
hôtels et les villas) est patent. Il suffit d'observer les fonds d'archives photographiques d'Héritage Métis pour s'en rendre compte. Le désir collectif du rivage se déploie à Métis à l'image de son éveil en Europe au milieu du XVIII' siècle et de son développement jusqu' au XIX ${ }^{e}$ siècle. La plage (The Beach) s'intègre à la riche fantasmagorie des lisières; elle s'oppose à la pathologie urbaine. Au bord de la mer. mieux qu'ailleurs, l'individu se confronte aux éléments, jouit de la sublimité du paysage, comme le souligne Alain Corbin (1988) dans son ouvrage Le territoire $d u$ vide.

En raison de leur localisation sur les plages, les grands hôtels de cette époque (aujourd'hui disparus) et les villas d'une architecture pluriellek ont participé à l'invention d'un paysage maritime où l'expérience au fleuve était manifeste (bains, baignade, voile côtière, piscine marine). En fait, Métis-sur-Mer (affublée à l'époque du nom de « Metis Beach \%) était développée suivant la transposition des modèles de villégiature balnéaire du sud de l'Angleterre. Ces stations maritimes, dès la première moitié du XVIII" siècle, proposaient des bains de mer, « des promenades en mer et surtout le yachting [... ]. Le bal, les salons de conversation et les salles de jeu permettent de passer d'agréables soirées ' (Corbin, 1988 : 288). Par contre, comme le souligne Corbin, le pittoresque marin ne semblait pas être l'interêt premier, tout au plus la frâtcheur des brises et l'effet tonifiant du bord de mer y sont évoqués. Lieu de thếâtralité sociale avant tout, à une échelle bien moindre, la villégiature de Métis-sur-Mer s"y est apparentée. La lecture des formes d'occupation de l'espace littoral dans son historicité révèle une communauté tournée vers un * hygiénisme hédonique s caractérisé par le bénéfique a grand air s du fleuve et le plaisir des lieux.

Avec la disparition des hôtels dans la deuxième moitié du $\mathrm{XX}$ ' siècle (après la Seconde Guerre mondiale)" balnéaire est partiellement occulté. La rive se privatise et se « végétalise » par l'appropriation des lots qui appartenaient autrefois aux grands hôtels. L'animation

sociale tourne le dos au fleuve par le jardin et le golf. Seule persiste la tradition de certains cocktails en fin de journée d'été que prenaient des habitants de Métis-sur-Mer sur les plages a vestiges $\$$ d'une époque révolue.

Dans le jardin des villas, le paysage est tout autre. Il n'est présent principalement que par les vistas (fenêtres) sur le fleuve. $\AA$ l'image des w hahas ${ }^{10}$, ces vistas étendent le jar-

din à l'infini dans le

fleuve, ce dernier étant l'espace de l'incer= nable, voire de l'incommensurable. Apparemment morne et abstrait, le fleuve est tout autre. Il est à la fois évocateur d'imaginaire ou d'un passé révolu (les baignades et la voile) et surtout de présence. C'est par ces vistas que l'air marin pénètre dans le jardin. Les courants d'air chaud et froid climatisent les jardins clos de Métis-sur-Mer. Ainsi, mềme si le fleuve ne se voit pas, sa présence est d'une prégnance apaisante par la sonorité du clapotis de l'eau et agaçante lors des grands vents. On le côtoie sans le toucher et c $c^{+}$est l'épaisseur de son air qui nous touche et qui pénètre la peau quand la brume tombe. C'est anssi par quelques-unes de ces vistas que l'on aperçoit les deux attraits signifícatifs de la communauté, soit le phare et les rochers des Boules ${ }^{\prime \prime}(«$ Bull $)$.

Malgré lạ présence des vistas sur le a fleuve/mer », les jardins privés de ce littoral sont avant tout des intériorités. Fermés à la vue du public de la rue par de grandes haies de cèdres, ils sont reclus sur eux-mêmes et ils interprètent le paysage terrestre (rocaille avec des pierres de l'arrière-pays, petite cascade, verger, etc.). Miniaturisation de scènes rupestres et sylvestres, plusieurs d'entre eux expriment l'art de l'ornementation florale de l'époque victorienne (style $\approx$ Gardenesque ${ }^{12}$ ) et de l'influence du paysagiste américain Jackson Downing (1815-1852). Jardins composites qui font avant tout usage d'arbres, d'arbustes, et de fleur's exotiques (par exemple, les Jardins de Métis), ils expriment le plein. Ils sont des condensés qui s'opposent à l'univers dilaté du a fleuve/ mer $\%$. Ils traduisent aussi la rencontre contrastée du sauvage (le Wilderness ${ }^{13}$ ) et l'extrême sophistication d'une idée de nature. Le * fleuve/mer * ne nous livret-il pas, dans sa condition actuelle, l'idée de plus en plus accentuée de cette première nature, une nature mythique sublimée à l'image des transcendantalistes américains du XIX" siècle ? Les phoques côtoient de plus en plus le littoral et les rumeurs 10cales laissent entendre le passage de quelques baleines bleues. Ainsi, cette villégiature littorale dont l'âme semble être le jardin crée une interface des natures. Du paysage balnéaire du début du siècle dernier, l'actuel paysage littoral de Métis-sur= Mer semble tirer son essence de cette singularité et la plénitude paysagère y est effective en tous points.

\section{L'habité littoral ; persistance du pays et inflexion au paysage}

L habitat linéaire du littoral, représentatif des régions du Bas-Saint-Laurent et de la Gaspésie et illustré avec éloquence par le segment du littoral qui s'étend de SainteFlavie à Grand-Métis, constitue un héritage de l'époque de la pêche. En effet, la pêche a été surtout un mode de vie, de même qu'un élément structurant l'occupation d'un espace caractérisé par une dispersion de l'habitat conforme à l'éparpillement des ressources (Dugas, 1981 : 80). Aujourd'hui, 
la prolifération de petits villages le long des rives demeure, en dépit de la disparition quasi complète des pêcheurs côtiers. Malgré ce relatif délin, le paysage demeure marqué par cette histoire et, un peu partout, on tente de sauvegarder les éléments typiques de la peche côtière.

Pour plusieurs ruraux, ceux des régions isolées en particulier, qui ont « trimé dur $*$ toute une vie sur des terres rocheuses et dans des chantier's de butcherons, le patrimoine bâti ou les paysages traditionnels peuvent représenter un passé révolu, un passé difficile. Comme tout le monde, ils veulent être * modernes $*$, se faire bâtir des maisons neuves, acquérir un mobilier au gout du jout. Voici ce qu'ecrivait Marcel Rioux dans les années 1960 sur le changement des valeurs dans l'Est du Québec :

Les plus vieux qui se souviennent des $\alpha$ annees noires ", comme ils disent, ef qui voient awjourd hui lears fils condaire une voiture, se batir une maison neuve et festoyer les samedis soirs ne sont pas très enclins à defendre leur epoque; d'autant plus que c'est ce nouveau genre de vie qui lear a apporté les pensions de vieillesse. Ils sont donc les premiers à maudire le passé. l...J Qui donc défendrait le passé si les plus vieux n'y tiennem pas? [...] Ce qui sort du magasin devient le nec plus ultra de la beauté et de la qualite (Rioux, 1961: 67).

Néanmoins s'il y a un aspect dụ passé auquel semblent tenir les populations locales, c'est bien la répartition linéaire de l'habitat au bord du littoral. Bien que les industries régionales et le développement du secteur des services contribuent à une certaine concentration de l'habitat, la distribution spatiale de la population baslaurentienne est restée sensiblement la même avec un attachement particulier au fleuve, qui est aujourd hui apprécié plus. pour son paysage que pour les ressources qu'on en retire. Qui plus est, l'implantation et l'agrandissement de grosses usines de transformation du papier à Matane auraient entraîné plus de constructions dans les petites localités environnantes que dans cette ville. Quand il n'émigre pas à l'extérieur de la région, le cultivateur ou le pêcheur de l'Est du Québec qui a changé d'occupation continue de demeurer dans le milieu qui lui est familier et où il s'est enraciné. Les travailleurs de cette région préferent se déplacer quotidiennement ver's leur lieu de travail plutôt que de déménąger.

Le choix de s'implanter sur le littoral constitue une caractéristique de l'aménagement du littoral métissien et ce, en dépit de l'évolution des significations qu'a pu prendre le fleuve depuis 1830. Même si, à partir de la deuxième moitié du XXe siècle, le fleuve ait pu, en effet, représenter l'étendue d'eau qui sépare les familles, le désir d"habiter le littoral ne s'est jamais démenti. Le type d'implantation qu'on y trouve, par son style vernaculaire, montre que ce désir correspond à une réalité du e pays s : le fleuve constitue la plus grande des arrière-cours. Les maisons s"inscrivent dans le lieu, Les façades sur route toument le dos au fleuve (au vent dominant, aux embruns). Ce type d'implantation, ancré dạns la réalité littorale, exposé à tous les regards, témoigne d'une volonté a d'être dedans $*$ et « d'être là $\%$.
A priori, une telle implantation ne témoigne pas du tout de la mise à distance nécessaire à la production de paysages. Pourtant, en s'exposant à la vue, l'habitat du littoral métissien participe parfois aux scènes paysagères. Le glissement du « pays $*$ au « paysage $*$ s'opere de plus en plus actuellement, L'habitat se «paysage * à la manière de l'art indiscipliné. Les habitantspaysagistes proposent des mises en scène qui font preuve d'une a artialisation $\aleph^{14}$ évidente de leur façon d'habiter le littoral. Un exemple frappant en est la procession de sculptures de béton qui émergent périodiquement au gré du flux et du reflux des marées au centre d'art Marcel Gagnon de Sainte-Flavie.

L'evolution de l'habitat du littoral, passant du « pays » au « paysage s en vertu d'un changement dans les représentations que les habitants se font de leur littoral habite, témoigne d'une certaine permanence des formes spatiales et, surtout, d'une mutation profonde du regard que posent les habitants sur eux-mêmes et sur leur littoral. Le littoral est devenu un espace où l'on peut créer de nouvelles significations qui puisent aux registres artistiques et ludiques. Cette mutation est sûrement en lien avec

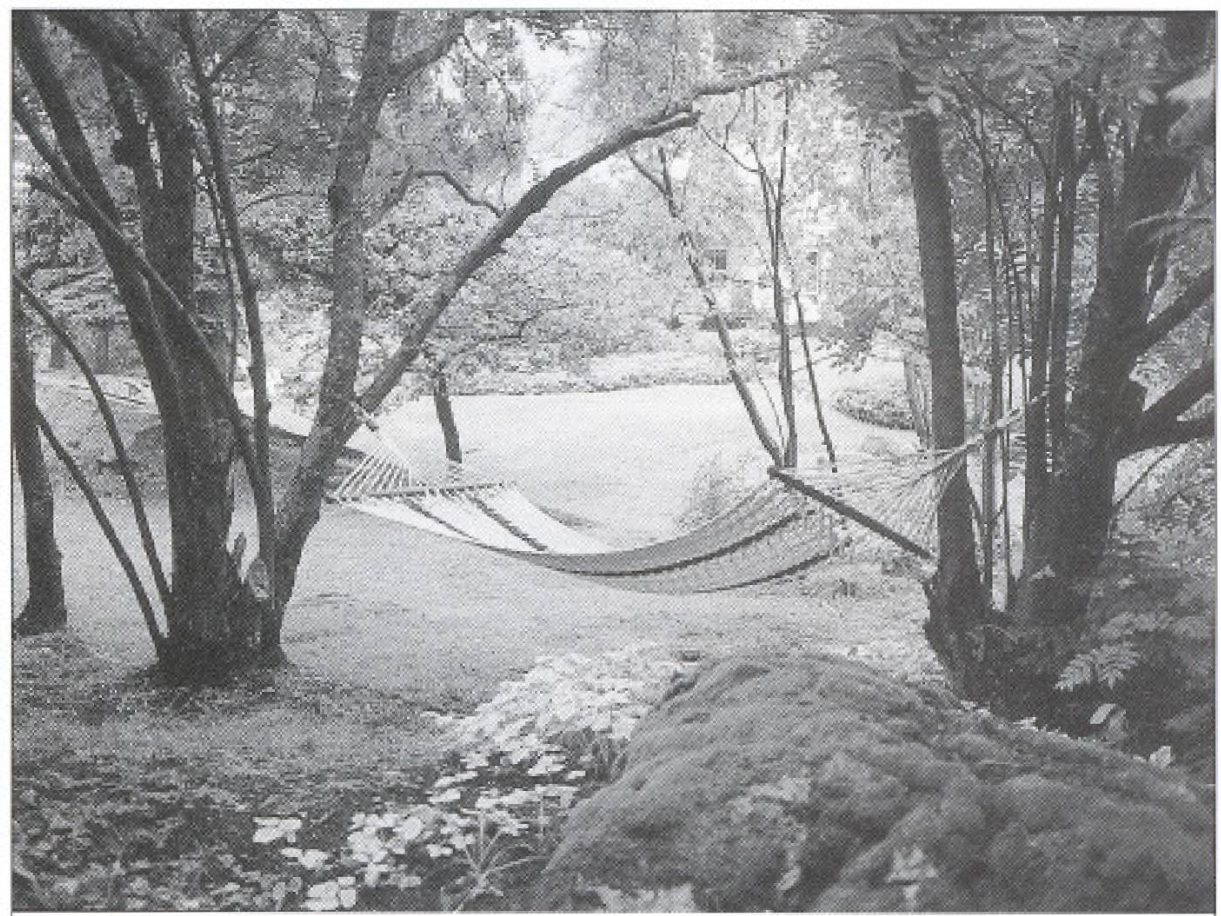

Intimité de jardin à Métis-sur-Mer.

Photo: Philippe PoulloovecGonidec, 1999. 


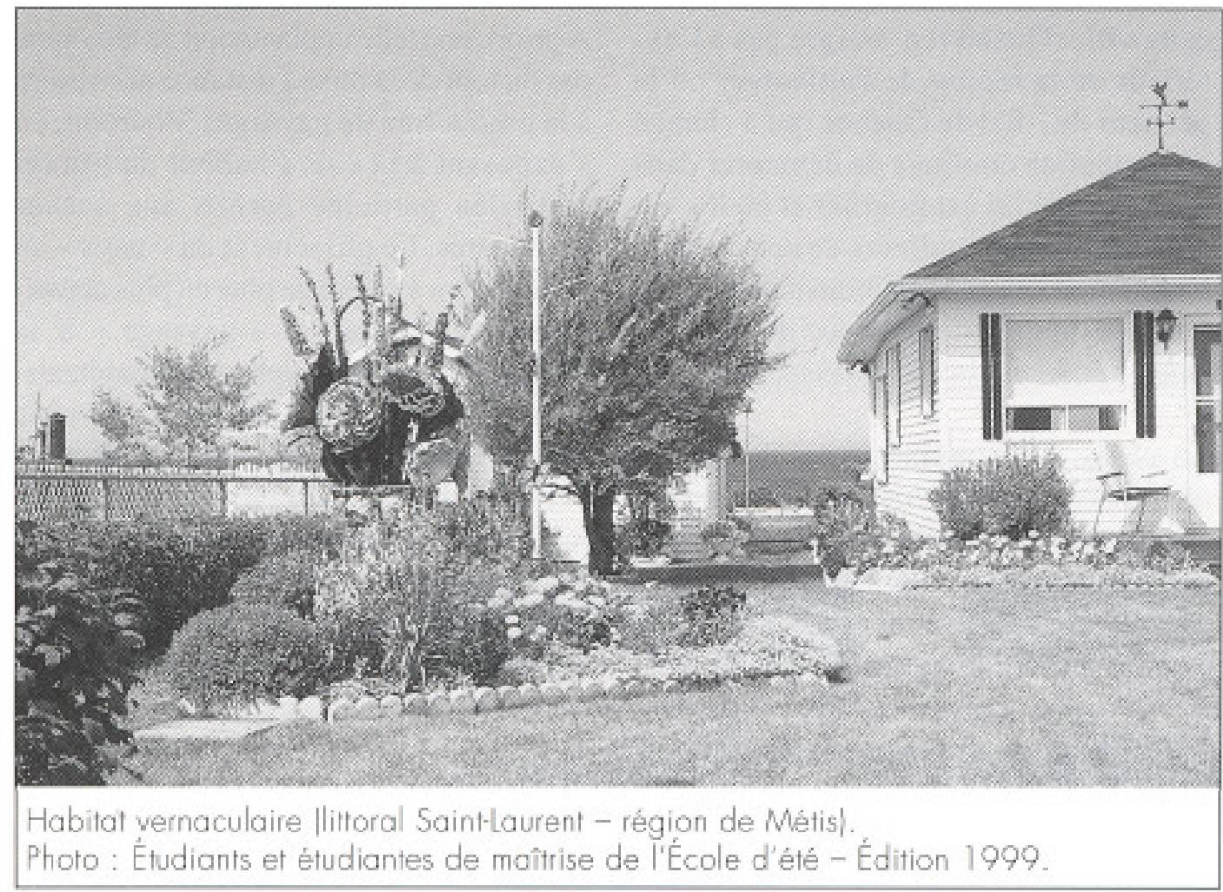

le croisement des regards exogènes et endogènes. En effet, il ne faut pas oublier que plusieur's bas-laurentiens d'origine resient attachés à leur littoral et, lors de leur retour temporaire ou permanent, posent dorénavant un regard où se mêlent l'ancrage au lieu et la curiosité détachée du visiteur.

\section{Les enjeux de paysage du littoral métissien}

Les mutations des formes, des pratiques et du regard représentent autant de facteurs qui participent à l'émergence d'un enjeu de paysage. Cet enjeu semble s'esquisser autant par le regard exogène des villégiateurs que par le regard endogène de la population locale. Il soulève la question de la reconnaissance des significations plurielles inhérentes au fleuve et de leur utilisation au sein de stratégie de requalification des espaces littoraux.

Ainsi, en regard de certaines actions développées, toutes semblent converger vers des stratégies de réinterprétation de ce que le pays a eté. Les actions portent ou convergent toutes vers la résurgence d'une mémoire locale ou régionale. A titre d'exemple, mentionnons le musée de Pointe-auPère sur le naufrage de 1'Empress of Ireland, le CISA (Centre d'interprétation du saumon de l'Atlantique) sur la rivière
Mitis, le Centre d' interprétation de l'aventure basque à Trois-Pistoles. En fait, toutes des initiatives liées à l'esthétique de la disparition (Poullaouec-Gonidec, 1993), aux regards nostalgiques ou résurgents.

Or, il semble que cette voie soit extrềmement limitée sur le plan des possibilités et sur le caractère distinctif de son développement. La récente initiative de la Société d'aide au développement de la collectivité de « La Métis s, par le biais du programme * Les collections numérisées du Canada * d'Industrie Canada qui vient de réaliser un site Internet sur le « Patrimoine Mitis ${ }^{15}$, ne semble pas laisser entrevoir une nouvelle approche de mise en valeur des attraits régionaux. L'inventaire qui est fait est des plus traditionnels. Le patrimoine est lié principalement aux biens matériels classiques (monuments historiques cités, patrimoine religieux, maisons d'époque, transports, patrimoine industriel et artisanal, patrimoine naturel et autres). A titre indicatif, le paysage n'y est cité qu'une seule fois pour vanter la multitude de petits lacs qui agrémente le territoire de SaintJoseph-de-Lepage. L'invention du paysage ne peut pas se satisfaire d'une simple mise en exposition de reliques passées.

Sans occulter les actions ou les projets développés dans ce sens, il est essentiel de redéployer la mise en valeur du littoral et sa qualification paysagère en fonction d'autres desseins, soit en les axant sur les sensibilités mêmes du lieu. Ne faut-il pas saisir les forces du littoral actuel ? $\mathrm{Ce}$ ressaiisissement passe par la capacité d'évoquer des imaginaires pluriels. Il impose done de nouvelles lectures d"un * rivage/ lieu * de tous les départs et de toutes les arrivées. $\mathrm{N}^{*} \mathrm{a}-\mathrm{t}-\mathrm{on}$ pas là une belle allégorie à l'invention paysagère du * fleuve/mer * ?

\section{L'observatoire des paysages, une instrumentation contributive à l'invention des singularités du territoire}

En réponse à cet enjeu et au défi qu'il représente, on peut entrevoir une actionclé en matière de reconnaissance des paysages littoraux et de la sensibilisation à ceux-ci. Cette piste pourrait prendre appui sur un projet d'observatoire des paysages de la région de Métis ${ }^{15}$. Ce projet se situe dans le prolongement des travaux de recherche menés â la Chaire sur le suivi des paysages au Québec et dans la foulée de la constitution des fonds d'archives d'Héritage Métis et des Jardins de Mếtis. Il s'agit de mettre en interface trois regards, ceux de l'artiste, de l'expert et du public. Ce projet intègre trois volets interdépendants:

1-Une mission photographique avec quelques photographes (volet a artiste $*$ ) pour recueillir des points de vue cadrés de territoire de la région rurale de Métis susceptibles de révéler des valeurs paysagères ${ }^{17}$.

2-Un volet instrumental d'observation des paysages (volet a expert $*$ ) et des territoires de la région de Métis, développé dans le cadre de l'école d'été de Métis ${ }^{18}$ avec les étudiants de maitrise de la Faculté de l'aménagement de l" Université de Montréal. Durant la semaine d'activités du mois d'août prochàin, les êtudiants seront amenés à définir les protocoles d'observation des paysages (les modalités en fonction des objectifs d'observation, les séquences d'observation et leur récurrence, etc.), à caractériser les points à observer, à valider leur 
pertinence sur le plan de la mise en valeur du territoire et à prendre les photographies nécessaires. On enwisage de poursuivre le suivi photographique annuellement lors des éditions subséquentes de l'école d'été de Métis, afin de saisir les transformations significatives et d'évaluer leur effet de levier pour la mise en valeur et le développement de la région.

3-Un volet observatoire \& grand public s qui sera l'occasion de demander aux visiteurs (touristes) des Jardins de Métis et à la population locale de prendre des photographies de la région et de les remettre aux responsables des Jardins pour constituer une banque d'inages (et évaluer la récurrence des sujets et des points de vue captés par l'objectif). Ce volet doit donner lieu à un événement public, soit celui de créer une journée de la photo du paysage de lạ région de La Métis (et de statuer sur le paysage de l'année...).

Ainsi, conçu sous cet angle, le projet d'observatoire ${ }^{19}$ est tres novateur puisqu'il réunit les trois principaux regards qui participent à l'invention ou la \& fabrication $*$ des paysages occidentaux depuis plus d'un siècle. Les défis de ce projet sont de susciter des questionnements sur le développement du territoire littoral, d'aider la population et les acteurs du milieu à prendre conscience de la valeur paysagère de leur milieu (et des phénomènes de mutation, de déqualification et de qualification) et de créer la distanciation nécessaire pour laisser émerger l'invention et la réinvention. Cette action, limitếe pour l'instant au cadrage visuel du territoire, devrait contribuer, au cours des années, à l'émergence de sensibilités occultées. délaissées et méconnues.

\section{Conclusion}

La quête du sens d'un milieu est le paysage. "Le paysage est une fiction, le paysage est une diction. /... / Construction culturelle, consiruction sociale, il désigne a la limite tout autre chose que ce que l'on a devant soi. Il est quasi-prétente en somme tant il autorise des échappées, tant il prend des libertés étonnantes avec les catégories ordinaires (Vilbrod, 1999: 327). Tel qu'esquissé précédemment, le paysage marin et littoral du pays métissien est significatif de ce constat. Toutefois, l'apparence d'un premier regard ( $\alpha$ le grand désert bleu $*$ ) ne doit pas fixer le dessein d'un territoire. Lieu des finitudes de la mer et de la terre, le littoral serait-il trop soumis à l' hégémonie visuelle, au simple regard ou a la souveraineté de la vue ${ }^{20}$ ? Trop souvent occultée par láa quête du spectaculaire, l'invention du paysage littoral n'est limitée en fait qu'à notre capacité d'êoquer. Ne devrions-nous pas fermer les yeux quelques instants pour laisser entrer quelques fabulations ? Ne faudrait-il pas réapprendre les temps de ce paysage? Ne fandrait-il pas délaisser quelque peu l' instantanéité du regard pour la lenteur d"un récit et le côtoiement persistant pour exalter ses formes imaginaires?

\section{Philippe Poullaouec-Gonidec}

est titulaire de la Chaire en paysage et environnement de l'Université de Montráal et professeur titulaire à l'École d'architecure de paysage de cette même institution.

\section{François Tremblay est chercheur}

a la Chaire en paysage et environnement de l'Université de Montréal et professeur invié à l'École d'architecure de paysage de cette mêne institution.

\section{Christiane Montpetit est anthro.}

pologue et chercheure à la Chaire en paysage et environnement de l'Université de Montréal.

\section{Notes}

1 Voir la reproduction dans $L$ invention de la liberte (Starobinski, 1987), tableau de la collection du musée Calvet à Avignon (France)

2 La relation esthétique des populations envers un espace vécu ou un espace frequenté prend appui sur la valcur esthétique que l'on attribue à un espace sur la base de certains modèles ou schemes culturels. Or, les modèles ou canons $*$ esthetiques et les attentes paysagères qu'ils projettent sont intimement liés à l'activité qu'ils déploient sur cet espace.

3 Lappartenance de l' individu à un groupe de référence lui fournit une connaissance specifique (des cles d'interprétation) qui, par la combinaison de divers médiums de distanciation au territoire (littérature, peinture, photographic, carte postale etc.). lui permet de médiatiser son experience intime au lieu. Le partage de cette experience mediatisée fait en sorte que se re. groupent des a communartés esthétiques * qui développent une relation d'engagement esthétique avec leur cadre de vie ou grâce à leurs activités.

4 Lire à ce sujet le travail d'un groupe d'êtudiants de maîtrise en paysage de la Faculté de l'aménagement de l'Université de Montréal dans le cadre de l'École d'êté de Métis en 1999, Le pays métissien, Parc régional éclaté desterrasses a la mer, de la mer aux terrasses, L. Beaucage, $\mathrm{K}$. Desjardins, M.C. Lemelin, N. Leonard, G. Paré, et M.B. Pasquier.

5 Ce terme renvoie aux propos d'Alain Roger sur l'imvention de la Montagne et de la Mer at sickcle des Lumières rassembles dans son texte Esthétique du paysage au sidele des Lumieres (1989:61-82). L'emploi du terme * invention s renvole à la creation du paysage dans les représentations collectives. Le paysage n'etant pas de l'ordre du a donné w, l'auteur décrit les processus par lesquels se sont effectuées ces fabrications in visu de paysages de montagne et de mer.

6 Sur le plan géographique, le littoral çvoqué se réfere à la région Est du Bas-SaintLaurent situće aux portes de la Gaspésie. Cette région est accessible par une route touristique littorale (Route 132) qui fait le a grand tour * de la Gaspésie. Le village de Sainte-Flavie (à $32 \mathrm{~km}$ à l'est de Rimouski) constitue les points de départ et d'arrivée de cette boucle routière fréquentee durant la periode estivale. A titre indicatif, * Les Jardins de Métis * accotés à la route 132 à Grand-Metis (a quelques kilomètres de Mont-Joli vers le nond-est au confluent de la rivière de la Mitis et du fleuve Saint-Laurent) attirent plus de 130000 visiteurs sur une période de quatre mois.

7 Ces propos sur les phénomènes d'invention du paysage de Métis-sur-Mer et de sa réinvention par le jardin sont le fruit d'une analyse qui repose sur une seric d'observations et de relevés photographiques colligés dans le cadre des recherches sur le suivi des paysages de la région (réf. : SMVP - Chaire en paysage et environnement) et de l'École d"êté de Métis (volet 
* Art des jardins et du paysage *, sous la direction de Philippe Poullaonec-Gonidec).

8 L'architecture de ces villégiatures datant de la fin du XIXe siècle est le conglomerat des styles néo-gothique, second empire, néo-Reine Anne, shingle set vernaculaire victorien. Woir à ce sujet le mémoire de mâtrise de Hyde et Zambrano (1991).

9 Le dernier hôtel fut détruit dans les annés 1970.

10 Ouverture pratiquée dans un mur de clôture pour prolonger ou pour ouvrir une perspective qui n'était pas conçue pour donner accès au paysage, mais pour eréter une surprise. Elle est généralement doublée d'un a saut-de-loup * (large fossé au-dewant du haha) qui permet d'avancer visuellement le jardin dans la campagne (voir Michel Conan, Dictionnaire historique de l'art des jardins, Editions Hazan). Dans le cas des jardins de Métis-sur-Mer, les escarpements rocheux du littoral en contrebas des jardins erént celle situation de a sautde-loup k.

11 Rochers situés en face de Métis-sur-Mer et contigus au village de Les Boules. Le toponyme du village vient de $\alpha$ boulders $*$, grosses roches abandonnes par les glaciers et polies par les vagues, souvent déplacées par le mouvement de ces dernières.

12 Mot anglais cree en 1832 par $J_{1}-\mathrm{C}_{\mathrm{a}}$ Loudon, à partir de a garden $s$, sur le modele de picturesque (voir Michel Conan, Dictionnaire historique de l'art des jardins, Editions Hazan).

13 Premiêre nature, woir ả ce sujet la théorie des trois natures de l'historien Dixon-Hunt (1996), ainsi que le livre d'Oelschlaeger (1991).

14 Terme reactual isé à propos du paysage par le philosophe Alain Roger (1999: 46). * Artialisation $*$ : $\alpha$ Processus artistique qui transforme et embellit la nature, soit directement (is sia), soit indirectement (in visu), au moyen de modeles $\%$. L'idée d'une « nature artialisée s apparaît chez Montaigne (Essais, III, * Sur les vers de Virgile *). Elle est reprise allusivement par Lalo (1912) dans son Introduction a l'esthétique. Alain Roger reprend le terme * artialisation s en lui apposant un statut nominal et dynamique dans deux ouvrages: Nus et paysages (1978) et Countraité du paysage (1997).

15 Le site Internet * Patrimoine Mitis \$ (httpy follections.ic.gc.ca/mitis) présente une collection photographique numerise agrementée de brèves fiches explicatives sur 1e patrimoine bâti (et naturel) des dix-neuf municipalités de la MRC de La Mitis.
16 Projet initié conjointement par Les jardins de Métis et la Chaire en paysage et environnement de l'Université de Montréal, en 1999, suite al l'elaboration du a Systeme de monitoring visuel des paysages $*$ (SMVP) en 1998, en collaboration avec HydroQuébec, les ministères de la Culture et des Communications, des Ressources naturelles, de l'Environnement et de la Faune, des Affaires municipales et Tourisme Québec.

17 A l'exemple des photos de Notman au debut des annés 1900 et de l'enquête photographique du peintre Paul-Ëmile Borduas, pendant l'êté 1938 (voir catalogue photographique, Un regard sur Pence).

18 Cette école d'été, initiée (en 1998) par la Chaire en paysage et environnement et l'École d'architecture de paysage de l'Université de Montréal conjointement avec les Jardins de Métis, rassemble à la fin de chaque étć (août) à Grand-Métis des étudiants et des étudiantes autour de l'enseignement de l'art des jardins et des paysages.

19 Liexemple le plus significatif dans ce domaine est l'observatoire des paysages français développé en 1989 par le ministère de I'Environnement qui fait appel à des artistes-photographes. L'objectif de cet observatoire est de détecter les changements qualitatifs que connaissent les paysages (et les territoires) au fil des ans, pour alerter les pouvoirs publies et leur permettre de prendre des mesures correctives.

20 Lire à ce propos l'article d'Augoyard $(1991 ; 51-59)$.

\section{Bibliographie}

Augoyard, Jean-François (1991), \& La vue, estelle souveraine dans l'esthétique du paysage ? *, * Au-delà du paysage moderne $\%$, in Le debat, ni" 65, p. 51-59.

Baricco, Alessandro (1998), Ocean mer, Editions Albin Michel.

Beaudet, Gérard, et Serge Gagnon (1999), * Esquisse d'une géographie structurale du tourisme et de la villegiature : l'exemple du Quebec in Cazelais Normand, Roger Nadeau, et Gérard Beaudet (dir.), Lespace touristique, Sainte-Foy, Les Presses de l'Université du Québec:

Berleant, Arnold (1997), Living in the Landscape - Toward an Aesthetics of Environnent, Lawrence, The University Press of Kansas.

Corbin, Alain (1988), Le territoire du vide, Paris, Editions Champs Flammarion.

Dixon-Hunt, John (1996), L'arr du jardin et son histoire, Editons Odile Jacabs.
Dugas, Clermont (1981), Un pays de distance et de dispersion, Sillery, Les Presses de l'Université du Québec.

Fortin, Jean-Charles, Antonio Lechasseur, Yvan Morin, Fernand Harvey, Jacques Lemay, et Yves Tremblay (1993), Histoire du Bas-Saint-Laurent, Coll. Les régions du Québec, $\mathbf{n}^{\circ} 5$, SainteFoy, Institut québécois de recherche sur la culture.

Hyde, Cynthia, et Gustavo Zambrano (1991), Merts-sur-Mer : conservation el developpement d'un village de villegiature du Bas-SaintLaurent, Mémoire de maîtrise, Université McGill, 1991.

Lalo, Charles (1912), Introduction à l'esthétique, Paris, Armand Colin.

Oelschlaeger, Max (1991), The Idea of Widemess, Yale Univerty Press.

Poullanouc-Gonidec, Philippe (1993), a Esthetique des paysages de la modernité $\%$, Le projel de paysage au Québec, in Trames, Revue de l'améragement, 9, p. 29-34.

Poullaouec-Gonidec, Philippe, Michel Gariépy, François Tremblay, Bernard Saint-Denis, Christiane Montpetit, et Julie Tellier (1999), Balisage des enjeux de payage concenant trois filieres de production d'electricite, Rapport de recherche de la Chaire en paysage et environnement, Montréal, Université de Montréal.

Rioux, Marcel (1961), Belle-Anse, Canada, Ministère du Nord canadien et des ressources nationales, Muscée national du Canada, Bulletin $n^{\circ} 138, n^{\circ} 37$ de la serie anthropologique.

Roger, Alain (1999), La mownance, cinquante mots pour le paysage, Editions de la Villette, 1999.

Roger, Alain (1997), Court traité du paysage, Editions Gallimard, Paris.

Roger, Alain (1989), Esthétigne du paysage au siècle des Lumieres, in Composer le paysage, Constructions et crises de lespace - 1789. 1992, Editions Champ Vallon.

Roger, Alain (1978), Nus et paysages, Edtions Aubier, Paris.

Starobinski, Jean (1987), L'invention de la liberte, Edition Skira, Flammarion.

Tittley, Louis (1995), * Les croisieres sur le Saint-Laurent... un peu d'histoire $*$, Téoros, 14(2), P. 12-14.

Vilbrod, Alain (1999), « Vue imprenable sur la mer , in La fabrication du paysage, Éditions Centre de recherche Bretonne et eltique, p. 325-335.

White, Kenneth (1997), Les rives du silence, Paris, Edition du Mercure de France. 